\title{
Minimum Jerk for human catching movements in 3D
}

\author{
Nadine Fligge \\ Joseph McIntyre \\ Patrick van der Smagt
}

\begin{abstract}
To investigate fast human reaching movements in 3D, we asked 11 right-handed persons to catch a tennis ball while we tracked the movements of their arms. To ensure consistent trajectories of the ball, we used a catapult to throw the ball from three different positions. Tangential velocity profiles of the hand were in general bell-shaped and hand movements in 3D coincided with well known results for 2D point-to-point movements such as minimum jerk theory or the 2/3rd power law. Furthermore, two phases, consisting of fast reaching and slower fine movements at the end of hand placement could clearly be seen. The aim of this study was to find a way to generate human-like (catching) trajectories for a humanoid robot.
\end{abstract}

\section{INTRODUCTION}

Although there is an infinite number of possibilities to move the hand from an initial position to an arbitrary final position, arm movements performed by different humans tend to be similar. For example Lacquaniti et al. [14] investigated a power-law relationship between the tangential velocity of the hand and the curvature of the trajectory, such that the more the trajectory is curved, the lower the velocity. It is furthermore known that unconstrained point-topoint hand paths in planar Cartesian space are approximately straight, with bell-shaped tangential velocity profiles [20], [1], [6]. And a common basis for grasping is that the gesture can be subdivided in a sequence of steps. These steps include an initial fast movement towards the object; then, when the hand is close to the object, a slow fine movement occurs. At the same time, the hand is formed to match the geometry of the object and is then closed around it [9], [10]. Different attempts have been made to describe and generate human movements according to computational principles, most prominent being the minimum-jerk model [6], minimum (commanded) torque change model [30], [21] and minimum variance model [7]. See Todorov [29] for an overview.

Based on this knowledge, our aim was to find a way to generate human-like (catching) trajectories for a humanoid robot that is already able to catch a ball, but does not, as yet, imitate human-like trajectories [2]. The first robotic catching in 3D reaches back to 1991 and was done by Hove and Slotine [8]. They implemented a trajectory matching algorithm on a cable-driven arm with four degrees of freedom and three fingers. The trajectory-matching algorithm combined an observer with a varying-strength filter, an error estimator, and an initial motion algorithm with the aim to optimally match the ball's trajectory with the end-effector of the robot arm. Riley and Atkeson [26] worked with a 30-degree of freedom humanoid. They set out to generate human-like behavior for virtual robots and humanoids. For ball catching they used a look-up table of human catching trajectories in combination with optimisation. Because their robot did not have a hand, they caught the ball with a funnel; thus exact orienting of the end-effector was not required for grasping. Another approach to generate human-like trajectories is to teach the robot via teleoperation, as successfully realized by Campbell et al. [3]. Here we set out to further identify characteristics of human catching movements, in the context of current theories of human motor control. In the following we review those theories and then relate them to the catching trajectories measured for a group of human subjects.

\section{A. Models}

\section{Minimum Torque Change Model}

The minimum torque change model postulates that movement dynamics should be optimized so as to minimize the time derivative of joint torque

$$
C_{T}=\frac{1}{2} \int_{0}^{t_{f}} \sum_{i=1}^{n}\left(\frac{d z_{i}}{d t}\right)^{2} d t
$$

were $z_{i}$ is the torque on the $\mathrm{i}$-th muscle and $n$ the number of muscles. To compute the optimal trajectory, the dynamics equation of the musculoskeletal system must first be specified, which is difficult [30], [12].

\section{Minimum Variance Model}

Harris and Wolpert [7] assumed that the neural control signals are corrupted by noise whose variance increases with the size of the control signal and further proposed that in the presence of such signal-dependent noise, the shape of a trajectory is selected to minimize the variance of the final arm position. Under this model the cost function to be minimized across repeated movements is the positional variance summed over the post-movement period.

\section{Minimum Jerk Model}

In contrast to the above, the minimum-jerk hypothesis is a kinematic model, independent of the dynamics of the musculoskeletal system. Flash and Hogan [6] hypothesized that arm movements are planned in external, that is Cartesian, space and rely on an optimisation of the jerk of the hand's trajectory. Jerk is the rate of change of acceleration, hence the third derivation of position. According to Flash and Hogan, in order to describe a point-to-point movement in a given time frame $t_{f}$ in $2 \mathrm{D}$, the objective function to be minimised is the time integral 
of the square of the magnitude of jerk:

$$
C_{j}=\frac{1}{2} \int_{0}^{t_{f}}\left(\left(\frac{d^{3} x}{d t^{3}}\right)^{2}+\left(\frac{d^{3} y}{d t^{3}}\right)^{2}\right) d t .
$$

Assuming zero initial and final velocity and acceleration, this can be simplified to:

$$
\begin{aligned}
& x(t)=x_{0}+\left(x_{0}-x_{f}\right)\left(15 \epsilon^{4}-6 \epsilon^{5}-10 \epsilon^{3}\right) \\
& y(t)=y_{0}+\left(y_{0}-y_{f}\right)\left(15 \epsilon^{4}-6 \epsilon^{5}-10 \epsilon^{3}\right)
\end{aligned}
$$

where $x_{0}$ and $y_{0}$ are the coordinates for the initial hand position $(t=0), x_{f}$ and $y_{f}$ for the final hand position $\left(t=t_{f}\right)$ and $\epsilon=t / t_{f}$.

For $2 \mathrm{D}$ point to point movements, all three of these models predict trajectories that are very similar to those gathered in experiments with humans. This similarity of the approaches allows us, to concentrate on the minimum jerk model for matching our recorded data. This decision was made because although generalisation to the other models is straightforward, they are computationally more complex. For ease of calculation of human-like trajectories for a humanoid robot in realtime, minimum jerk complies best with the requirements. We will therefore concentrate on the minimum-jerk approach to model and predict human wrist movements in 3D. In the following we tracked hand trajectories of 11 subjects during single-handed catching and compared these data with trajectories generated with help of the minimum jerk model.

\section{B. Human Ball Catching}

Human ball catching is an excellent task for studying movements in three-dimensional space, because a defined start and end point can be seen and it is a natural movement with a time constraint. Many experiments have investigated human ball catching, but most of them considered only catching in 2D. For example, Kajikawa et al. [11] investigated differences between human hand trajectories depending on the fragility object to be caught. A robust object is caught after the catcher's hand reaches the interception point (IP). To catch a fragile object, the catching movement is divided in four phases: 1) straight approach to the object, 2) reversing the hand movement, 3) accelerating the hand to reduce the velocity error, 4) positioning the hand at the object and tracking it. This is done to reduce the impact of the fragile object in order to catch it without any damage. Others, like McIntyre et. al [18] and Senot et al. [28], have concentrated more on when the ball is to be caught, taking into account the influence of gravity on the target. Savelsbergh and colleagues have studied the predictive control of the hand by occluding the sight of the catcher for certain time frames [27], thus looking at the effect of varying temporal constraints on the organization of the ongoing movement [17], while Land et al. [13] have studied the sensory information used to drive the interceptive response by tracking the eyes of batsmen while hitting a fast bowler's ball in cricket. Dessing et al. [4] observed in their experiments that, even if the initial hand position of the human is the same as the predicted interception point, catchers typically first move their hand away from this position and then back again. They concluded that lateral hand movements in catching are influenced by prospective control tied to the ball's approach direction, instead of the prediction of its interception point.

Experiments in three dimensions are less common. Here studies have been carried out in large volumes, such as catching in baseball games [13], thus studying the strategies by which subject run to a location where the ball can be caught.

\section{MethodS}

To examine human movements during catching, 11 righthanded healthy subjects were tracked while catching a tennis ball. They were aged between 23 and 32, two of them female, with heights ranging from 161 to $193 \mathrm{~cm}$.

To ensure equal trajectories of the ball, a catapult was used to throw the ball from three different, randomly varying positions. The catcher was instructed to catch with the right hand only and to always place the hand at the same initial position (that is at his/her leg). He/she was instructed not to step forward or bend the knees. We wished to understand the subject's movement strategy based on on-line visual information about the ball's trajectory. Thus, they were not allowed to practice the task. Furthermore, to avoid the catcher getting used to the trajectory of the ball and in consequence optimise the catching strategy, the speed of the tennis ball throws varied in random order. A screen was fixed in front of the catapult to prevent the catcher from seeing how much the catapult was preloaded, thus preventing an a priori estimate of what the ball's velocity would be. The ball became visible to the catcher shortly after it left the catapult.

The experimental setup is shown in figure 1. The position of the catcher was marked at the floor. To reduce the occlusion of markers three cameras were used, consisting of the Stereo Camera System smARTtrack (cam1 and cam2) and a third camera, ARTtrack2 (cam3). The smARTtrack was positioned at the right hand side in front of the catcher and the ARTtrack2 was positioned at the right hand side behind the catcher. Tracking frequency was $60 \mathrm{~Hz}$. A video camera was placed to the left of the catcher. The origin of the room coordinate system was defined as the tip of the left foot of the catcher. The $x$-axis points in the direction of the catapult, $y$-axis to the right and z-axis upward. Elbow and wrist of the catcher were each equipped with a cluster of 4 markers, while a single marker was positioned at the catcher's shoulder. The ball was equipped with nine flat markers, to ensure that at least one marker was seen from the cameras for every time step. The measurement accuracy of the ball was thus $6.5 \mathrm{~cm}$ due to its diameter. The catapult was placed $3.60 \mathrm{~m}$ in front of the catcher and changed between three different positions during the experiment. These positions were chosen exactly in front of the catcher (pos1), to the right (pos2) and to the far right (pos3).

\section{Data analysis}

In total, every catcher executed 40 trials, 21 of which had the same speed of the ball and were thus analyzed. Throws 


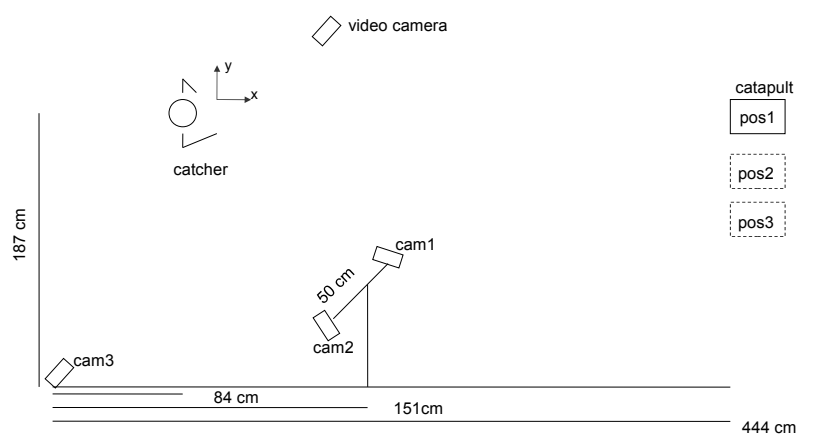

Fig. 1. Experimental setup from above. Cameras are located at the ceiling. $\mathrm{X}$ direction is in direction of the flying ball, $\mathrm{y}$ direction is in the frontal plane of the subject, $\mathrm{z}$ direction is height

with other speeds were either to slow or to fast to allow accurate catching. A total of 231 data sets were collected, 177 of which were usable while 54 contained too few data points due to occlusions. Hand and ball trajectories were plotted in 3D and in 2D over time (see figure 2). Hand trajectories were numerically differentiated to calculate movement speed and acceleration of the hand. The video sequences of the hand were used to investigate the interception point (IP), that is the point in time when the ball was caught. A 3D extension to the $2 \mathrm{D}$ minimum jerk model introduced above has been developed (see appendix for details) to compare with the natural human catching movements.

\section{RESULTS}

As described for movements in 2D [6], the movements for ball catching in 3D were quite smooth, as shown in Figure 2. The trajectories were quite similar for each individual catcher but they differed from catcher to catcher. Ten persons out of eleven produced similar trajectories for every trial, one person produced quite different movements from trial to trial.

Additionally to analysis of the video tapes, the IP can be estimated in the plots from the ball's trajectory and the hand trajectory. One can see there happens to be a point of minimal velocity (PMV) before the IP. Tangential velocity profiles showed a distinct PMV for 151 of 177 trials and were in general bell-shaped up until this point. As seen in Figure 2, PMV almost always occurred before interception; only in 4 trials did PMV and IP take place at the same time.

Mean duration from PMV to IP was 0.06 seconds. Distance from PMV to IP varied from 10 to $88 \mathrm{~mm}$. In the 87 trials where we could measure the hand trajectory between PMV and IP, (the others being excluded due to occlusions) we observed that the 3D trajectories following PMV looked quite different for every catcher. Three different strategies could be seen for this phase of the catching movement:

- go towards the ball on its trajectory

- follow the trajectory of the ball downwards

- do some kind of sidewards (correction) movement

Most catchers executed the same strategy in this respect every time, but two performed markedly different movements for the different catapult positions.
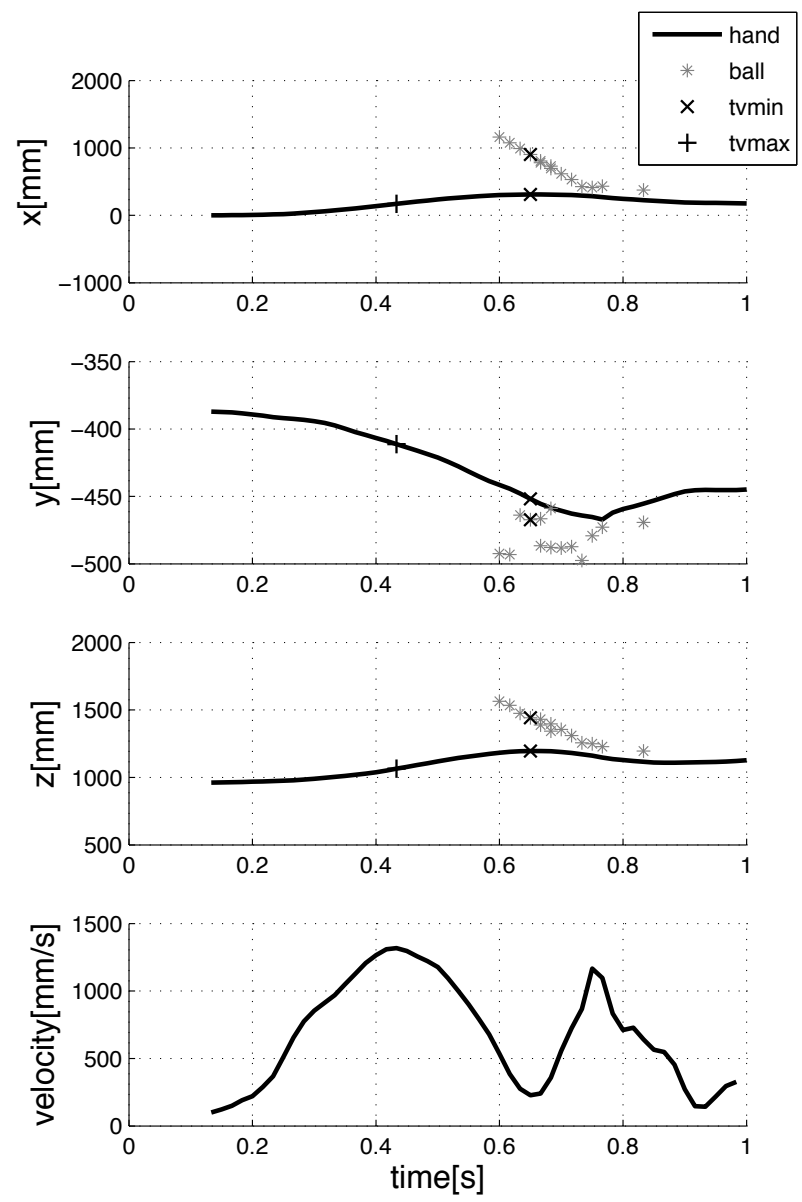

Fig. 2. Example of data of the catching movement for one person. Marked are the point of minimal velocity (PMV or rather tvmin) and the point of maximal velocity (tvmax). $\mathrm{X}$ direction is in direction of the flying ball, $\mathrm{y}$ direction is in the frontal plane of the subject, $\mathrm{z}$ direction is height. Due to the fact, that the diameter of the ball is about $6.5 \mathrm{~cm}$, markers vary a bit in y direction

\section{Minimum jerk in $3 D$}

Minimum jerk for point-to-point movements predicts a straight-line movement when the boundary conditions are such that initial and final velocity and acceleration are zero. Due to the non-zero velocity and acceleration at PMV, the generated minimum jerk trajectories may be curved. We calculated 3D minimum jerk trajectories only for the first part of the movement (start to PMV), the movement from PMV to IP being too short to provide a meaningful comparison between model and data. Velocity and acceleration of the hand were estimated for the start of the movement and the point of minimal velocity (PMV). Minimum jerk trajectories were then calculated for each trial, using these constraints, and plotted against their respective measured data.

To judge the quality of fitting, the residual error was computed as area between the human hand trajectory and minimum jerk trajectory. In $74 \%$ of the trials, minimum jerk fitting was judged to be adequate, meaning that the integrated error was smaller than $0.005 \mathrm{~m}^{2}$. This threshold was chosen from visual inspection of the results where a 
clear cut between errors smaller than $0.005 \mathrm{~m}^{2}$ or larger than $0.007 \mathrm{~m}^{2}$ was observed.
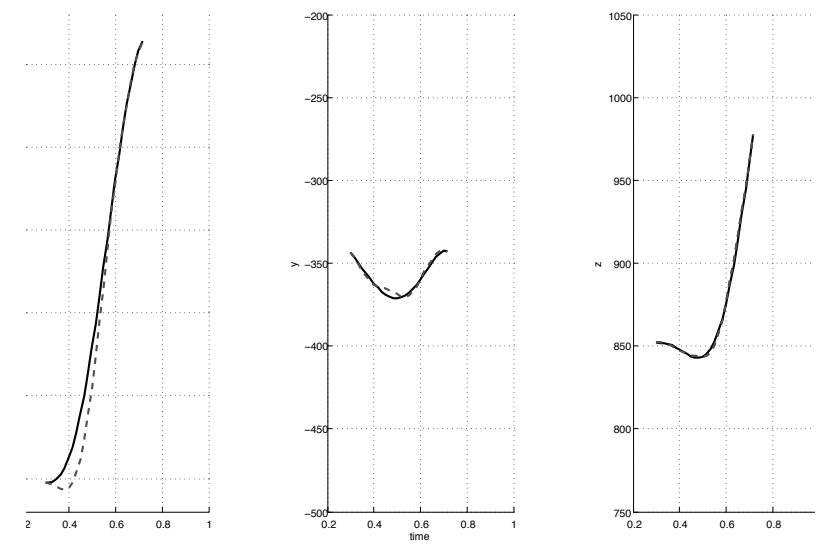

Fig. 3. Comparison of minimum jerk generated trajectory (line) and real hand movements (dashed)
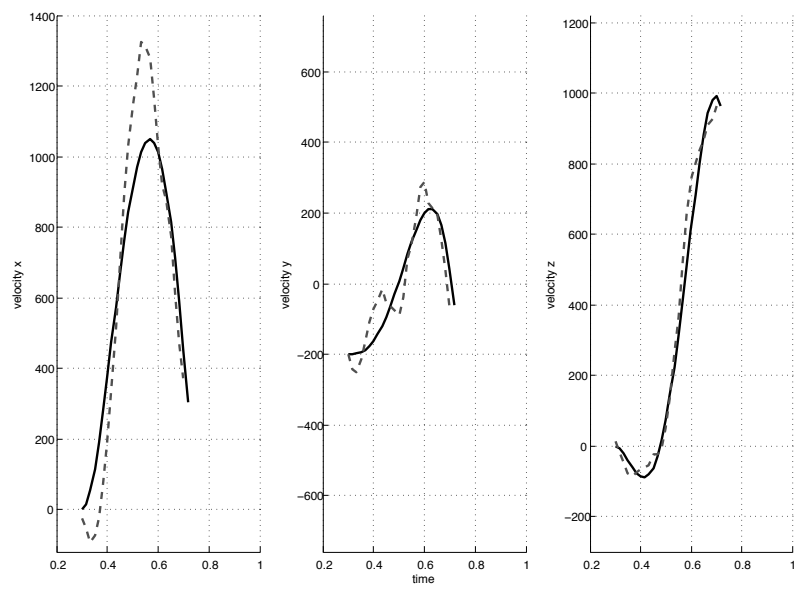

Fig. 4. Comparison of minimum jerk generated trajectory (line) and real hand velocities (dashed)

\section{Discussion}

Proposals for catching strategies can be divided into two broad classes: predictive and prospective [31]. Predictive strategies assume that the catcher computes an a priori estimate of where the ball will go, selects an interception point along the ball's trajectory and then plans and executes a movement of the arm so as to place the hand at the selected interception point at the right time. The predicted interception point may be updated over time as new information about the target's trajectory is acquired, but the predictive strategy basically assumes that the movement has a defined endpoint that is computed some finite time before the arrival of the ball or the hand at that interception point.

Prospective strategies are, on the other hand, based on realtime calculations of how to direct the hand in such a way that it should intercept the ball at some time in the future, without any advance estimate of where the interception will occur [31]. A simple example of such a law is used in boating. If, while standing on one ship one observes another ship on

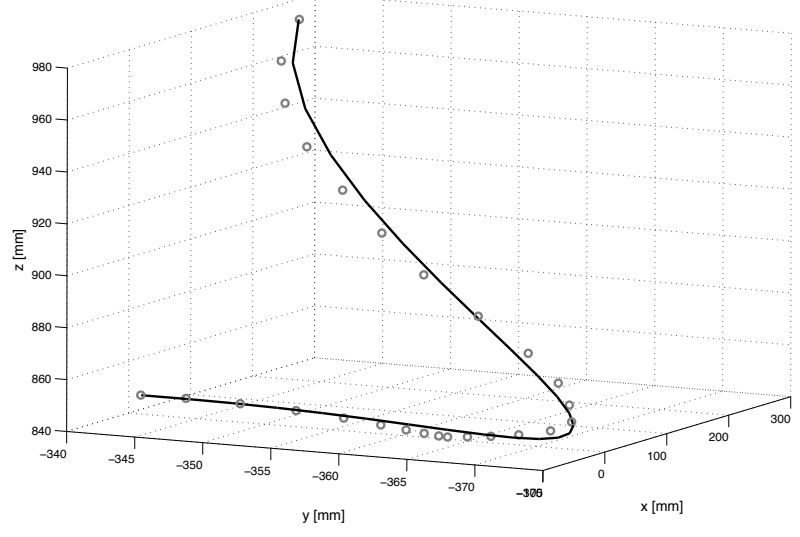

Fig. 5. Comparison of minimum jerk generated trajectory (line) and real hand trajectory (dots) in 3D

the horizon at a particular azimuth with respect the current heading, and if after a period of time in which the boats move forward along constant heading the azimuth remains the same, one can be assured that the two boats are on a collision course. To intercept the ball in a soccer match one could run forward while adjusting the speed such that the azimuth of the ball with respect to the player remains constant [5]. Similar strategies can be used to run forward or backwards on the playing field to catch a fly ball [16]. A cogent example is the work of Peper et al. [24] in which a control law that depends on the estimated time-to-contact (TTC) and the instantaneous difference between the hand and the target's positions are used to determine the instantaneous velocity of the hand. By following this law, the hand will be progressively driven along a path that assures interception.

The observation of a well-defined PMV, in most cases, is in line with the two-phase movements described by Jeannerod [9] for reaching and grasping, applied here to the dynamical case of moving to intercept a moving target. The catcher would initiate a movement that is largely preprogrammed to a position close to the desired interception point. The subject could then switch to a more prospective, feedback-based control law [23] to drive the second phase of the movement so as to correct for errors in the estimated trajectory of the ball or to satisfy additional constraints such as minimizing the relative velocity between the ball and the hand. Note that the initial transport phase of the movement need not be fully terminated before the second movement phase is initiated. Thus, the hand velocity need not fall to zero between movement phases. The observed non-zero PMV is consistent with the superposition of two component sub-movements that overlap in time [22], [15].

The bell-shaped velocity profile up to the PMV argues for a predictive mechanism in the programming of the initial movement. Movement reversal have been observed for a particular prospective strategy when applied to oblique movements [19], but for the straight-on ball movements used here a purely prospective strategy should produce a monotonic trajectory of the hand until it reaches the interception point. Deceleration of the hand also differentiates the observed 
human behavior from the interception strategy implemented by Hove and Slotine[8] in which the robot was programmed to try and match the velocity of the flying object; since the flying ball accelerates under the influence of gravity, velocity of the hand would, under that strategy, continually increase until the catch is made. A predictive strategy that drives the hand toward a predetermined interception point, coupled with a subsequent corrective movement, is much more consistent with the point of minimal velocities reported here.

The fact that the initial movement follows the minimum jerk criteria is further evidence, since this would mean that initial movement is programmed according to the same laws that govern other point-to-point movements [6], [14], although dynamical optimisation cannot be discounted as well [21]. In this respect, the catching movements that we observed reflect the principles adopted by Riley et al. [25] for their humanoid robot, but they state that their interception point was gradually updated as new visual information comes available. Thus, one would not expect their algorithm to produce the PMV and second movement component that we observed here. The nature of the second sub-movement (predictive or prospective) is less well defined by the results of this experiment, but the predictive nature of the first movement seems quite clear. The different directions of hand movements during PMV and IP suggest two possible interpretations of what the catcher does after PMV. On the one hand, he or she could either estimate the IP at the very beginning of the throw (as described in [13]), go there as accurately as possible and then look at the ball again to perform a corrective movement to the true interception point. Alternatively, one might move rapidly to PMV and then attempt to follow the ball in order to match its velocity, similarly to what has been observed when catching fragile objects [11]. In our experiments, this strategy may be intended to reduce the impact of the ball hitting the hand, rather of saving a fragile object.

In summary, kinematic analysis suggests that humans use predictive strategies to catch a ball, coupled with a final, corrective sub-movement just prior to contact, applying rules similar to those used for point-to-point and reach-to-grasp movements of the hand. Refining robotic control strategies to reflect these observations could lead to a better understanding of the human behavior, and perhaps more robust robot performance.

\section{REFERENCES}

[1] C. Atkeson and J. Hollerbach, Kinematic features of unrestrained vertical arm movements, The Journal of neuroscience, Vol. 5(9), pp. 2318-23330, Sept. 1985

[2] O. Birbach, U. Frese, B. Bauml, Realtime perception for catching a flying ball with a mobile humanoid, IEEE International Conference on Robotics and Automation, pp.5955-5962, May 2001

[3] C. Campbell, R. Peters, R. Bodenheimer, W. Bluethman, E. Huber, R. Ambrose, Superposition of behaviors learned through teleoperation, IEEE Transactions on Robotics, Vol. 22(1), pp. 79-90, 2006

[4] J.C. Dessing, C. E. Peper, D. Bullock, P.J. Beek, How position, velocity and temporal information combine in the prospective control of catching: data and model, Journal of Cognitive Neuroscience 17(4), pp. 668-686, April 2005

[5] B. Fajen, W. Warren, Visual guidance of intercepting a moving target on foot, Perception, Vol. 33(6), pp.689-715, 2004
[6] T. Flash, N. Hogan, The coordination of arm movements: an experimentally confirmed mathematical model, Journal of Neuroscience Vol. 5(7), pp. 1688-1703, 1985

[7] Ch. Harris and D. Wolpert, Signal-dependent noise determines motor planning, Nature, Vol. 394, pp. 780-784, August 1998

[8] B. Hove, J. Slotine, Experiments in robot catching, Proc. IEEE American control conference, pp.380-385, June 1991

[9] Jeannerod M., The neural and behavioural organization of goal directed movements. Clarendon Press, Oxford, 1988

[10] M. Jeannerod, M. A. Arbib, G. Rizzolatti, H. Sakata, Grasping objects: the cortical mechanisms of visuomotor transformation, Trends Neuroscience Vol. 18, pp.314-320, 1995

[11] S. Kajikawa, M. Saito, K. Ohba, H. Inooka, Analysis of human arm movement for catching a moving object, Proc. IEEE Conference on System Man and Cybernetics, pp. 698-703, 1999

[12] Y. Kaneko, E. Nakano, R. Osu, Y. Wada, M. Kawato, Trajectory formation based on the minimum commanded torque change model using the Euler-Poisson Equation, Systems and Computers in Japan Vol. 36 (2), pp.92-103, 2005

[13] M. Land, P. McLeod, From eye movements to actions: how batsmen hit the ball, Nature Neuroscience Vol. 13(7), pp.1340-1345, 2000

[14] F. Lacquaniti, C. Terzuolo, P. Viviani, The law relating kinematic and figural aspects of drawing movements, Acta Psychologica Vol. 54, pp.115-130, 1983

[15] D. Lee, N. Port, A. Georgopoulos. Manual interception of moving targets. II. On-line control of overlapping submovements, Experimental Brain Research, Vol. 116(3), pp.421-33, Oct 1997

[16] M. McBeath, D. Shaffer, M. Kaiser, How baseball outfielders determine where to run to catch fly balls, Science, New Series. Vol. 268(5210), pp.569-573, Apr 1995

[17] L. Mazyn, G. Montagne, G. Savelsbergh, M. Lenoir, Reorganization of catching coordination under varying temporal constraints, Motor Control, Vol. 10, pp. 143-159, 2006

[18] J. McIntyre, M. Zago, A. Berthoz, F. Lacquaniti, Does the brain model Newton's law?, Nature Neuroscience Vol. 4(7), pp. 693-694, July 2001

[19] G. Montagne, M. Laurent, A. Durey, R. Bootsma, Movement reversals in ball catching, Experimental Brain Research, Vol. 129, pp 87-92, 1999

[20] P. Morasso, Spatial control of arm movements, Experimental Brain Research, Vol. 42(2), pp. 223-227, April 1981

[21] E. Nakano, H. Imamizu, R. Osu, Y. Uno, H. Gomi, T. Yoshioka, M. Kawato, Quantitative examinations of internal representations for arm trajectory planning: minimum commanded torque change model, Journal of Neurophysiology, Vol. 81, pp.2140-2155, 1999

[22] K. Novak, L. Miller, J. Houk, The use of overlapping submovements in the control of rapid hand movements, Experimental Brain Research, Vol. 144(3), pp.351-64, June 2002

[23] H. Okuda, H. Takeuchi, S. Inagaki, T. Suzuki, S. Hayakawa, Understanding of positioning skill based on feedforward feedback switched dynamical model, Proc. IEEE Conference on Intelligent Robots and Systems, pp. 3057-3057, 2009

[24] L. Peper, R. Bootsma, D. Mestre, F. Bakker, Human Perception and Performance, Journal of Experimental Psychology, Vol. 20(3), pp.591612, June 1994

[25] M. Riley, A. Ude, C. Atkeson, Methods for Motion Generation and Interaction with a Humanoid Robot: Case Studies of Dancing and Catching, GVU Technical Report, Georgia Institute of Technology, 2000

[26] M. Riley, C. Atkeson, Robot catching: Towards engaging humanhumanoid interaction, Autonomous Robots, Vol. 12 pp.119-128, 2002

[27] G. Savelsbergh, H. Whiting, J. Pijpers, A van Santvoord, The visual guidance of catching, Experimental Brain Research, Vol. 93 (1), pp.148-156, February 1993

[28] P. Senot, M. Zago, F. Lacquaniti, J. McIntyre, Anticipating the effects of gravity when intercepting moving objects: differentiating up and down based on nonvisual cues, Journal of Neurophysiology, Vol. 94, pp. 4471-4480, December 2004

[29] E. Todorov, Optimality principles in sensorimotor control, Nature Neuroscience, Vol.7 (9), pp.907-915, Sept 2004

[30] Y. Uno, M. Kawato, R. Suzuki, Formation and control of optimal trajectory in human multijoint arm movement, Biological Cybernetics, Vol. 61, pp. 89-101, 1989

[31] W. Warren, Action mode and laws of control for the visual guidance of action In: Meijer OG, Roth K (eds) Complex movement behavior: 
'The' motor-action controversy. North-Holland, Amsterdam, 1988, pp.339-380

\section{APPENDIX}

Based on equation (2) the cost function for minimum jerk in $3 \mathrm{D}$ can be written as

$$
C_{j}=\frac{1}{2} \int_{0}^{t_{f}}\left(\left(\frac{d^{3} x}{d t^{3}}\right)^{2}+\left(\frac{d^{3} y}{d t^{3}}\right)^{2}+\left(\frac{d^{3} z}{d t^{3}}\right)^{2}\right) d t
$$

But unlike Flash's and Hogan's [6] boundary conditions that set velocity and acceleration to zero at the end of the movement, they are not zero at the point of minimal velocity (PMV). Hence Equation (3) is not a solution in this case. The general solution of the cost function is a polynomial of fifth order for each dimension. For $\mathrm{X}$ this gives:

$$
x(t)=a_{0}+a_{1} t+a_{2} t_{2}+a_{3} t_{3}+a_{4} t_{4}+a_{5} t_{5}
$$

The coefficients of this polynomial are calculated with respect to the initial and final position, velocity and acceleration in $x, y$ and $z$. For initial conditions in $\mathrm{x}, x_{0}, \dot{x}_{0}$ and $\ddot{x}_{0}$ and final conditions $x_{t_{f}}, \dot{x}_{t_{f}}$ and $\ddot{x}_{t_{f}}$, one gets:

$$
\begin{aligned}
a_{0} & =x_{0} \\
a_{1} & =\dot{x}_{0} \\
a_{2} & =\frac{1}{2} \ddot{x}_{0} \\
a_{3} & =-\frac{10}{d^{3}} x_{0}-\frac{6}{d^{2}} \dot{x}_{0}-\frac{3}{2 d} \ddot{x}_{0}+\frac{10}{d^{3}} x_{t_{f}}-\frac{4}{d^{2}} \dot{x}_{t_{f}}+\frac{1}{2 d} \ddot{x}_{t_{f}} \\
a_{4} & =\frac{15}{d^{4}} x_{0}+\frac{8}{d^{3}} \dot{x}_{0}+\frac{3}{2 d^{2}} \ddot{x}_{0}-\frac{15}{d^{4}} x_{t_{f}}+\frac{7}{d^{3}} \dot{x}_{t_{f}}-\frac{1}{d^{2}} \ddot{x}_{t_{f}} \\
a_{5} & =-\frac{6}{d^{5}} x_{0}-\frac{3}{d^{4}} \dot{x}_{0}-\frac{1}{2 d^{3}} \ddot{x}_{0}+\frac{6}{d^{5}} x_{t_{f}}-\frac{3}{d^{4}} \dot{x}_{t_{f}}+\frac{1}{2 d^{3}} \ddot{x}_{t_{f}}
\end{aligned}
$$

where $d$ is the duration of the movement from start to PMV.

The same equations apply to $\mathrm{Y}$ and $\mathrm{Z}$. 